\title{
Die Progression wird nicht gebremst
}

Fragestellung: Kann durch die Gabe von intravenös verabreichten Immunglobulinen (IVIG) die Progression der Erkrankung bei Patienten mit leichter bis mittelschwerer Demenz vom Alzheimer-Typ beeinflusst werden?

Hintergrund: Daten aus Pilotstudien konnten einen Effekt von IVIG auf Biomarker im Liquor in dieser Patientengruppe zeigen. Eine Studie mit 24 Patienten postulierte auch eine Besserung der Kognition (gemessen mit dem MMSE) nach sechs Monaten und Stabilisierung nach 18 Monaten.

Patienten und Methodik: In dieser placebokontrollierten, doppelblinden Phase-III-Studie wurden 390 Patienten mit leichter bis mittelschwerer Alzhei-

Relkin NR, Thomas RG, Rissmann RA et al. A phase 3 trial of IV immunoglobulin for Alzheimer disease. Neurology 2017; 88: $1768-75$ mer-Demenz randomisiert und erhielten über 18 Monate 0,2 oder 0,4 g pro kg Körpergewicht IVIG alle zwei Wochen. Als primäre Endpunkte wurden die Veränderun- gen der kognitiven Funktion sowie der funktionellen Leistungsfähigkeit nach 18 Monaten ab Baseline angenommen. Daten zur Sicherheit sowie Daten zu Biomarkern wurden von allen Patienten im Rahmen der Studie erhoben.

Ergebnisse: Es konnte kein Effekt auf die beiden untersuchten Endpunkte in der Studie nachgewiesen werden. Eine signifikante Veränderung fand sich in der Auswertung der Plasma- $\beta$ Amyloidspiegel $\left(\mathrm{A} \beta_{1-42}\right.$; nicht für $\left.\mathrm{A} \beta_{1-40}\right)$. Die Untersuchung zur Sicherheit zeigte keinen Unterschied zwischen IVIG und placebobehandelten Patienten bezüglich des Auftretens von amyloidassoziierten Veränderungen in der Bildgebung. IVIG-behandelte Patienten hatten häufiger systemische Reaktionen, aber weniger Lungeninfekte als d iejenigen, die Placebo erhielten.

Schlussfolgerungen: In der Studie konnte kein positiver Effekt auf die Kognition oder die funktionelle Leistungsfähigkeit von Patienten im Stadium der leichten bis mittelschweren Demenz nach Gabe von IVIG nachgewiesen werden. IVIG werden in der untersuchten Dosierung gut vertragen.

\section{- Kommentar von Richard Dodel, Essen}

\section{Keine Effekte auf Kognition oder funktionelle Leistungsfähigkeit}

Die Rationale für den IVIG-Einsatz bei Patienten mit Demenz vom Alzheimer-Typ basiert unter anderem auf dem Nachweis natürlich vorkommender Autoantikörper [1], die nicht durch Kontakt mit Fremdmolekülen gebildet werden, sondern seit der Geburt vorhanden sind und veränderte körpereigene Bausteine aufspüren sowie zu deren Metabolisierung beitragen können. Sie werden durch spezifische B-Zellen gebildet und stellen etwa zwei Drittel des im Körper verfügbaren Antikörperpools. Kürzlich konnten natürlich vorkommende Autoantikörper gegen $\beta$-Amyloid und Tau nachgewiesen werden, die bei der Erkrankung erniedrigt sind. Nachdem in fünf Studien mit sechs bis 24 Patienten nach IVIG-Gabe ein Effekt auf Biomarker oder kognitive Parameter nachgewiesen wurde, erfolgten eine sechsmonatige Phase-II-Studie [2] und die jetzt vorliegende Studie über 18 Monate. In dieser sehr sorgfältig geplanten und durchgeführten Studie konnte weder auf den kognitiven noch auf den funktionellen Endpunkt ein positiver Effekt versus Placebo gezeigt werden. Dies galt auch für leicht

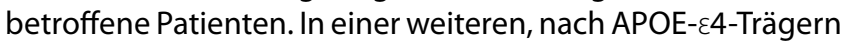
stratifizierten Subgruppenanalyse zeigte sich ein signifikanter Unterschied im MMSE für mit 0,4g/kg KG IVIG behandelte Patienten. Diese Studie reiht sich in die Studien mit experimentellen Therapieansätzen ein, die im Stadium der AlzheimerKrankheit ihre vordefinierten Endpunkte nicht erreichten. Eine rege Diskussion über das Scheitern der unterschiedlich ausgerichteten Substanzen haben eine Vielzahl von Gründen in den Raum gestellt und mündete in die Biomarker-Kaskaden-Hypothese, die besagt, dass Biomarker ein unterschiedlich zeitliches
Auftreten im Krankheitsverlauf haben und im klinischen Stadium der Erkrankung eine Beeinflussung dieser Marker „zu spät “ ist [3]. Inzwischen wird empfohlen, krankheitsmodifizierende Substanzen nicht im klinischen Stadium der Demenz zu testen, sondern im präklinischen Erkrankungsstadium, also bei noch kognitiv gesunden Personen. Dies bedeutet aber auch, dass keine krankheitsmodifizierenden Medikamente mehr für diese Patientengruppe entwickelt werden. Große Hoffnungen werden aber in diese präventiven Studien gesetzt; erste Ergebnisse sind jedoch nicht vor 2019/2020 zu erwarten. Es wäre hoch interessant, die Wirkung von IVIG bei dieser Population im präklinischen Stadium der Demenz zu untersuchen.
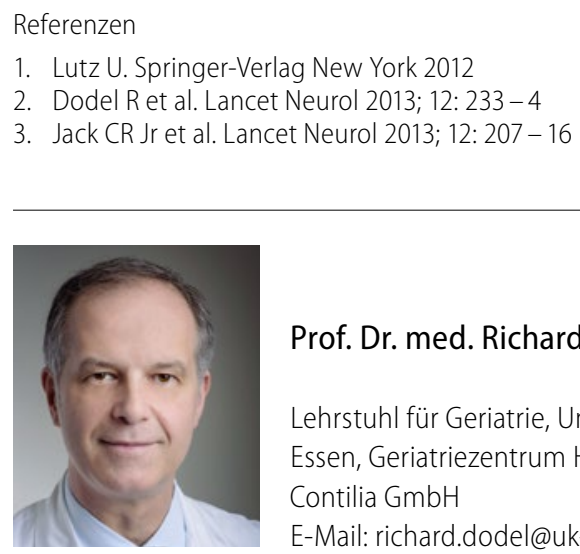

Prof. Dr. med. Richard Dodel, Essen

Lehrstuhl für Geriatrie, Universitätsklinikum

Essen, Geriatriezentrum Haus Berge,

Contilia GmbH

E-Mail: richard.dodel@uk-essen.de 